\title{
Bassa prevalenza di utillizzo della DP in Italia: è questo il problema?
}

\author{
Mario Salomone
}

\author{
Unità Operativa Aziendale, Nefrologia e Dialisi \\ Azienda Ospedaliera CTO - CRF - M. Adelaide, Torino
}

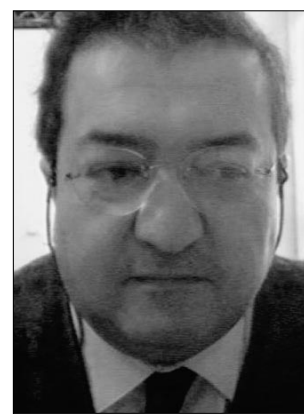

$\mathbf{L}$ e motivazioni che stanno alla base della bassa prevalenza di trattamento con dialisi peritoneale in Italia sono già state più volte discusse in questi anni. L'iniziativa di affrontare oggi nuovamente la questione mi ha lasciato perplesso: ho il timore che, posto in questi termini, questo non sia un argomento di interesse per la maggior parte dei nefrologi italiani e che il tutto si risolva in dotte ma altrettanto sterili parole. Proprio per questo ho titolato il mio intervento "Bassa prevalenza di utilizzo della DP in Italia: è questo il problema?". Ritengo infatti che la questione vera sia se il sistema dialitico attuale nel suo complesso, prese in considerazione le variabili e le variazioni socio-economiche a cui va incontro il nostro modello di sviluppo, possa continuare a garantire $\mathrm{i}$ buoni risultati che nel complesso ha finora dato. $\mathrm{E}$ se un uso più esteso della DP possa essere una delle risposte ma sicuramente non l'unica (basti pensare alla necessità dell'incremento della attività di trapianto renale).
Nonostante l'incremento del numero di pazienti in dialisi (con incidenze nell'ordine di 120 casi per milione di abitanti negli ultimi anni, ed elevata prevalenza di pazienti anziani e ad elevata comorbidità) il sistema ha finora retto l'urto e i risultati in termini clinici (sopravvivenza, mobilità e qualità del trattamento) sono buoni. La prevalenza media di pazienti in DP in Italia è al 12.98 (dati RIDT 1998) di circa il $10 \%$ e questa prevalenza è rimasta pressoché costante negli anni, con ampia variabilità da Regione a Regione (Tab. I). Non v'è dubbio che la distribuzione dell'uso della DP sia dipesa in larga parte oltre dall'interesse verso questa forma di terapia di alcuni Gruppi nefrologici anche dall'esistenza in alcune Regioni di un sistema sanitario prevalentemente o totalmente pubblico. La colonna destra della tabella riporta la distribuzione della percentuale di Centri pubblici nelle diverse Regioni e questa distribuzione ricalca abbastanza fedelmente l'impiego della metodica di dialisi peritoneale.

È evidente che il "privato" non ha interessi sufficienti a introdurre e sviluppare la DP. Assisteremo probabilmente, in un futuro non troppo lontano, a grossi sconvolgimenti in ambito sanitario e la componente "privata" acquisterà verosimilmente un peso sempre maggiore (introduzione della componente "no profit", liberalizzazione dell' accesso al privato ecc.). Nell'ambito della dialisi "privata" stiamo assistendo a una sua concentrazione diretta dalle stesse ditte del settore dialitico. Una concentrazione simile peraltro si sta attuando per le ditte che offrono materiali e servizi relativi alla DP. Non penso questi processi possano essere bloccati e debbano essere visti solamente in senso negativo. Penso piuttosto debbano essere attentamente osservati, regolamentati e guidati. In altre parole si dovrà andare probabilmente a una integrazione e collaborazione più stretta tra pubblico e privato e quest'ultimo avrà interesse a sviluppare il trattamento di DP, se i rimborsi saranno più adeguati e se il pubblico potrà offrire in qualche maniera, tutta da studiare, una fattiva e remunerata collaborazione. Le domande che dobbiamo porci mi sembrano quindi: possiamo far diventare la DP una forma "interessante" di trattamento per il privato? Quali risorse bisogna impegnare in questo processo? Quale ruolo attivo il Centro pubblico può avere in questo processo?

Alla luce delle variazioni epidemiologiche osservate in questi anni nella popolazione dialitica (incremento del numero di pazienti anziani e con condizioni di rischio clinico elevato) ci sono anche altre domande che 
dobbiamo porci: riusciremo almeno a mantenere percentuali di pazienti in trattamento di dialisi peritoneale analoghe alle attuali? Quale spazio deve essere riservato alla dialisi peritoneale automatizzata (APD)?

I dati epidemiologici in nostro possesso hanno sottolineato che la prevalenza della DP negli ultimi anni è stata mantenuta anche in relazione a un incremento della APD. La dialisi peritoneale automatizzata non solo ha dimostrato le possibilità di "recuperare" pazienti altrimenti da destinare al trattamento di dialisi extracorporea (pazienti di peso più elevato, che stanno contraendo la funzione renale residua) ma permette di inviare a domicilio pazienti anziani e solo parzialmente autosufficienti che abbiano un supporto familiare. Rimane tuttavia il problema, destinato a crescere soprattutto nelle aree metropolitane, dei pazienti anziani con scarso o nullo supporto familiare ("soli"), ad elevato grado di comorbidità. A questi pazienti è possibile offrire un trattamento di DP extraospedaliero solo se il paziente è "protetto" in un ricovero per anziani o in altra analoga struttura in cui sia attivato un processo di collaborazione per l'uso della dialisi peritoneale automatizzata. Anche qui il problema è al di fuori della clinica ed è prevalentemente politico.

Un altro quesito che riguarda da vicino la situazione italiana è quello relativo al rapporto tra bassa prevalenza di DP e basso numero di trapianti renali eseguiti. La risposta a questa domanda rappresenta, a mio parere, un aspetto non marginale del problema e pur non avendo i numeri e le competenze per affrontarla, ritengo che la scelta della DP sarebbe favorita (anche per pazienti più giovani) se la probabilità a breve termine di un trapianto fosse più elevata. Penso peraltro che da parte dei nefrologi che si occupano di dialisi peritoneale l'attenzione a garantire ai pazienti in DP l'accesso al trapianto renale dovrebbe essere migliorata. A esempio il paziente giovane che in DP va incontro a un importante sovrappeso nonostante un buon controllo di tutti gli altri parametri clinici deve essere

TABELLA I - PREVALENZA E INCIDENZA DI PAZIENTI IN DIALISI PERITONEALE IN ITALIA (DATI RIDT 1998); \% DI CENTRI PUBBLICI PER REGIONE

\begin{tabular}{lccc}
\hline & $\begin{array}{c}\text { \% DP in } \\
\text { trattamento } \\
\text { a fine anno }\end{array}$ & $\begin{array}{c}\text { \% DP primo } \\
\text { trattamento } \\
\text { nei nuovi } \\
\text { ingressi - 1998 }\end{array}$ & $\begin{array}{c}\text { \% Centri } \\
\text { pubblici }\end{array}$ \\
\hline PIEMONTE & 18.6 & 25.2 & 100 \\
VALLE D'AOSTA & 17.6 & 43.8 & 100 \\
LOMBARDIA & 18.4 & 28.3 & 93 \\
TRENTINO-ALTO ADIGE & $* *$ & $* *$ & 80 \\
VENETO & 14.8 & 24.8 & 100 \\
FRIULI-VENEZIA GIULIA & 12.7 & $* *$ & 100 \\
LIGURIA & 25.3 & 33.2 & 100 \\
EMILIA-ROMAGNA & 10.0 & 14.5 & 100 \\
TOSCANA & 10.6 & 14.2 & 100 \\
UMBRIA & 16.4 & 25.2 & 100 \\
MARCHE & 15.9 & 22.4 & 94 \\
ABRUZZI & 16.7 & 21.6 & 90 \\
LAZIO & 3.6 & 6.1 & 39 \\
MOLISE & 3.9 & 6.4 & 86 \\
PUGLIA/ BASILICATA & 7.6 & 11.7 & $70 / 83$ \\
CALABRIA & 15.8 & 23.0 & 97 \\
SICILIA & 1.2 & $* * *$ & 26 \\
SARDEGNA & 7.4 & 10.4 & 90 \\
CAMPANIA & $* * *$ & $* * *$ & 15 \\
\hline
\end{tabular}

*** dati non disponibili; **non possibile ricavare dati relativamente ai soli pazienti in DP

mantenuto in metodica o piuttosto avviato a un programma di extracorporea?

Sempre nell'ambito del "paziente più giovane" (considerando con questo termine i pazienti con età < ai 55-60 anni) è sicuramente da rivalutare la possibilità di un trattamento di emodialisi domiciliare, magari come naturale continuazione del trattamento di dialisi peritoneale domiciliare per il paziente che deve interrompere la DP.

Altri sarebbero ancora gli aspetti da considerare, di cui peraltro più volte si è discusso in questi anni: la difficoltà di strutturare un team medicoinfermieristico che si occupi della
DP, la programmazione del drop-out della dialisi peritoneale verso l'emodialisi, la qualità di vita, lo sviluppo di un trattamento domiciliare guidato dall'ospedale, ma che utilizzi le strutture sanitarie che operano sul territorio, e altri aspetti ancora.

I lavori che hanno dimostrato che la dialisi peritoneale è equiparabile alla dialisi extracorporea per quanto riguarda la mortalità e la morbilità sono molti; altrettanto dimostrato è che la DP ha un elevato drop-out verso l'emodialisi. Solo ultimamente si è finalmente iniziata a considerare la DP come programma integrato nel trattamento della uremia cronica e sono stati messi in risalto quegli 
aspetti positivi che possono far considerare la DP un ottimo trattamento, ancorché non estensibile a tutti i nuovi ingressi in dialisi, soprattutto nelle fasi iniziali del trattamento sostitutivo: rallentamento della progressione con mantenimento più a lungo della funzione renale residua, maggior possibilità di un incremento graduale e progressivo della dose di dialisi. In altre parole non si tratta di "privilegiare" una tecnica dialitica piuttosto che un'altra, ma di valutare come l'integrazione delle metodologie possa migliorare la quantità e la qualità della vita del paziente in uremia cronica. Sicuramente la possibilità di poter offrire al paziente in uremia terminale anche il trattamento di dialisi peritoneale deve essere valutato in una "economia di scala" facendo riferimento alle risorse finanziarie, metodologiche, culturali a nostra disposizione. Dal punto di vista dell'intervento si dovrebbe arrivare a un miglioramento delle compensazioni del trattamento con DP e con APD; procedere alla attivazione di strutture di ricovero per anziani in cui sia possibile attivare un programma di dialisi peritoneale automatizzato; stimolare le amministrazioni a investire nei confronti del trattamento domiciliare integrato, che dovrebbe essere possibile utilizzare in maniera più diretta da parte dei responsabili del Centro dialisi. Se vogliamo governare il fenomeno penso anche dovremmo riconsiderare il nostro intervento a partire dalle fasi più precoci dell'insufficienza renale cronica e cominciando di qui per il paziente l'educazione alla "collaborazione al proprio trattamento", stimolando anche le associazioni dei pazienti in questo sforzo.

salomone@libero.it 